\title{
Outcomes of total versus partial colectomy in fulminant Clostridium difficile colitis: a propensity matched analysis
}

Nasim Ahmed ${ }^{1,3^{*}}$ and Yen-Hong Kuo ${ }^{2}$

\begin{abstract}
Background: The Total Abdominal Colectomy (TAC) is the recommended procedure for Fulminant Clostridium Difficile Colitis (FCDC), however, occasionally, FCDC is also treated with partial colectomies. The purpose of the study was to identify the outcomes of partial colectomy in FCDC cases.

Method: The National Surgical Quality Improvement Program database was accessed and eligible patients from 2012 through 2016 were reviewed. Patients 18 years and older who were diagnosed with FCDC and who underwent colectomies were included in the study. Patients' demography, clinical characteristics, comorbidities, mortality, morbidities, length of hospital stay and discharge disposition were compared between the group who underwent partial colectomy and the group who underwent TAC. Univariate analysis followed by propensity matching was performed. A $P$ value of $<0.05$ is considered as statistically significant.

Results: Out of 491 patients who qualified for the study, 93 (18.9\%) patients underwent partial colectomy. The pair matched analysis showed no significant difference in patients' characteristics and comorbidities in the two groups. There was no significant difference found in mortality between the two groups ( $30.1 \%$ vs. $30.1 \%, P>0.99)$. There were no differences found in the median [95\% CI] hospital length of stay (LOS) (23 days [19-31] vs. 21 [17-25], $P=0.30$ ), post-operative complications (all $P>0.05$ ), and discharged disposition to home ( $33.8 \%$ vs. $43.1 \%$ ) or transfer to rehab ( 21.55 vs. $12.3 \%, P=0.357$ ) between the TAC and partial colectomy groups.
\end{abstract}

Conclusion: The overall 30 days mortality remains very high in FCDC. Partial colectomy did not increase risk of mortality or morbidities and LOS.

Level of evidence: Level IV.

Study type: Observational cohort.

Keywords: Fulminant Clostridium Difficile Colitis, Partial colectomy, Total colectomy, Mortality

\section{Introduction}

Fulminant Clostridium Difficile Colitis (FCDC) is one of the severe conditions of colon that is associated with very high mortality [1]. Approximately $4 \%$ of patients

*Correspondence: Nasim.Ahmed@hmhn.org

1 Division of Trauma \& Surgical Critical Care, Jersey Shore University Medical Center, 1945 State Route 33, Neptune, NJ 07754, USA

Full list of author information is available at the end of the article with Clostridium difficile colitis progress into fulminant course [2]. Aggressive resuscitation and early colectomy resulted in lower mortality $[3,4]$. Total abdominal colectomy (TAC) is a standardized procedure for FCDC [5].

Alternate to TAC, another procedure was proposed almost 10 years ago, loop ileostomy (LI) and colonic lavage [6]. This new procedure was compared to TAC but no significant difference in overall mortality was found in 
a small study including 10 patients of (LI) compared to 13 patients of TAC [7].

Recent studies from the NSQIP database showed colectomy is still the procedure of choice $[3,8]$ but as an alternative to TAC, studies suggest a partial colectomy which does not appear to increase mortality [8]. In addition, the partial colectomy could ensure that part of the colon can be saved thus minimizing the metabolic consequences that ensues with TAC [9]. The study that compared the partial colectomy with standardized total abdominal colectomy for FCDC adjusted the characteristics of patients on multivariable logistic regression analysis [8]. However, propensity score comparison methodology was reported to be a better mode of performing observational studies [10]. As a result, we decided to conduct a study using propensity score matching analysis to find out the outcomes of patients who underwent partial colectomy for FCDC.

\section{Methods}

All adult patients age 18 years and older who were diagnosed with Clostridium difficile colitis and underwent emergency colectomy for the indication of Clostridium difficile colitis were included in the study. The data came from the National Surgical Quality Improvement Program (NSQIP) database from the calendar years 20122016. The American College of Surgeons developed the NSQIP database for the improvement of outcomes in surgical patients [11]. More than 700 institutions across the US participate in the NSQIP. The Clostridium diffcile colitis defined as if the patient develops diarrhea with positive Clostridium difficile on laboratory test of stool by culture or PCR assay or Glutamate dehydrogenase EIA/ latex agglutination or cytotoxin test. Excluded from the study all emergency colectomies that were performed other than the indication of Clostridium difficile colitis. All elective colectomies were also excluded from the study.

We analyzed two groups, (partial colectomy [when a segment of the colon \{right sided or left sided\} was removed] versus total colectomy [where the entire colon was removed]), looking at gender, race, preoperative sepsis status, white blood cell counts, blood transfusion before the surgery and ventilator dependent respiratory failure prior to surgery, the American Society of Anesthesiologists (ASA) classification of the surgery, wound classification and comorbidities; history of diabetes mellitus (DM), smoking, chronic obstructive pulmonary disease (COPD), ascites, hypertension on medication (HTN), congestive heart failure, chronic renal failure (CRF), CRF on dialysis (CRF-D), disseminated cancer, steroid use. All comorbidities, sepsis and septic shock were defined as per NSQIP data dictionary.
The primary outcome of the study was 30 days all-cause mortality, while secondary outcomes were post-operative complications, hospital length of stay and discharge disposition.

\section{Statistics}

First, patient demographic information and outcomes were summarized using summary statistics (median with interquartile range (IQR) [first quartile-third quartile]) for continuous variables, and frequency and percentage for categorical variables). Then, the group that underwent total abdominal colectomy was compared with the group that underwent partial colectomy on patient's demography, diseases severity, comorbidities, and outcomes. The Wilcoxon Rank Sum test was used for continuous variables, and the Chi-square test was used for the categorical variables.

The propensity score for total abdominal colectomy was calculated for each patient and the one-to-one matching was performed using the "nearest neighbor" as the matching method to pair a subject who had TAC with the subject who underwent partial colectomy. The propensity score matching was performed using the $R$ package "MatchIt" [12]. The propensity scores were calculated using all the variables that may have impacted the decision to perform one procedure type versus other procedure type and that included (gender, age, race [white versus non-white], sepsis status, blood transfusion, respiratory failure, ASA class, wound class and all comorbidities mentioned above. After matching, the numeric and graphical diagnostics were used to evaluate the improvement in the variables. Again, summary statistics were performed as described above. One-toone comparison between the two matched groups was performed using Wilcoxon Signed Rank test for the continuous variables. The McNemar's test was used to compare the categorical variables between the two matched groups. If the level of a categorical variable was more than two, the Stuart-Maxwell test was used. For the total hospital length stay, the Kaplan-Meier procedure was used to estimate the median time, and the standard error was estimated using the Greenwood's formula. The logrank test was used to compare the time (Kaplan-Meier curves) between the two groups. The 2 -sided $P$ value was reported for each test. A $P$ value of $<0.05$ was considered an indication of statistical significance. Statistical analysis was performed using the $\mathrm{R}$ language [13].

\section{Results}

Patients' characteristics \& Univariate analysis

Out of 491 patients who qualified for the study, 398 (81.1\%) patients underwent total abdominal colectomy. Only 93 (18.9\%) patients underwent partial colectomy. 
Fifty one out of $93(54.83 \%)$ patients underwent right sided colectomy and remaining $42(45.17 \%)$ patients had left sided colectomy. Approximately $84 \%$ of patient underwent colectomy for toxic colon and approximately $16 \%$ of patients underwent colectomy for perforation. The median $[\mathrm{IQR}]$ age of the patient who underwent partial colectomy was 66 [55-75], the male and female distribution was almost split equally with slight increase of male dominance, $53 \%$ and about $77 \%$ of patients were Caucasians. There were significant differences found between the two groups, TAC and partial colectomy groups, regarding the presence of septic shock prior to surgery $(67.8 \%$ versus $52.7 \%, P=0.03)$. TAC group presented with higher percentage of life threatening of ASA class $(66.3 \%$ versus $59.1 \%, P=0.029)$ and found to have higher percentage of patients with history of steroid use (22.9\% versus $12.9 \%, P=0.047)$. Significantly higher proportion of patients in TAC group mounted severe leukocytosis $\left(\geq 20 \times 10^{9} / \mathrm{L}\right)($ Table 1$)$.

\section{Propensity matching analysis}

The propensity matching created 93 pairs. There was significant improvement in patients' characteristics after the matching. The pair matched analysis showed that all the differences between the two groups found in univariate analysis were balanced after the matching. (Figure) 1 .

There were no differences between the groups, TAC versus partial colectomy, regarding median age 65[5775 ] vs. 66 [55-75], race [Caucasians] $73.1 \%$ vs. $77.4 \%$, gender [male] (49.5\% vs. $52.7 \%)$, septic shock prior to surgery (55.9\% vs. $52.7 \%)$ and ventilator dependent respiratory failure (37.6\% vs. $29 \%)$ and comorbidities, all $P$ values were $>0.05$ (Table 2 ).

There was no significant difference in mortality between the TAC and partial colectomy groups (30.1\% vs. $30.1 \%, P>0.99)$. The median [95\% CI] hospital length of stay between the TAC and partial colectomy was (23 [19-31] vs. 21 [17-25], $P=0.30$ ). There was no significant difference found between the groups, TAC and partial colectomy, regarding the discharged disposition to home (33.8\% vs. $43.1 \%)$ or transfer to rehab (21.55 vs. $12.3 \%, P=0.357$ ) (Table 3).

There were no significance differences found between the two groups regarding surgical site infections, incidence of pneumonia, urinary tract infections, sepsis, septic shock, return to operating room, failure to wean from the ventilator and readmission rates Table 4.

\section{Discussion}

Our study showed that the majority, $81 \%$ of FCDC patients underwent total abdominal colectomy while only $\sim 19 \%$ of patients had partial colectomy. The allcause 30 -days mortality in the matched group was $30.1 \%$.
Partial colectomy did not show any difference in overall mortality or post-operative complications and discharge disposition to home.

Prior studies showed that early colectomy had a better survival probability than no colectomy $[4,14]$. Total abdominal colectomy has been the practice pattern for many decades in fulminant cases of FCDC [5]. In 2015, World Society of Emergency Surgery (WSES) recommendation was to perform early TAC in the management of FCDC [15]. The updated WSES guidelines in $2019 \mathrm{kept}$ the TAC as a primary choice of surgical intervention [16].

Very few prior studies have examined the comparison of mortality outcome of TAC with partial colectomy. A study examined the surgical mortality of the FCDC found that patients underwent partial colectomy had the worse outcome than the TAC [17]. The major limitation of the study was a very small sample size. The total number of patients included in the study was 14 and the major reasons for the high mortality in partial colectomy were not very clear. Byrn and colleagues examined 73 patients with FCDC who underwent colectomy [18]. Most colectomies $(86 \%)$ were subtotal colectomy, only 4 patient had right hemicolectomy and 5 had left hemicolectomy and one patient had total colectomy. One patient who had left hemicolectomy was converted to total colectomy. No significant difference was found in overall mortality whether the patient underwent partial colectomy or subtotal colectomy ( $10 \%$ vs $38 \%$, respectively; $P>0.05$ ). A recent NSQIP database study included all patients with FCDC who underwent colectomies from 2007 through 2015 [8]. The study consisted of 733 patients and found slightly higher mortality rate in partial colectomy group when compared to TAC ( $37.1 \%$ vs $34.7 \%, \mathrm{P}=0.58$ ) in univariate analysis. However, multiple logistic regression analysis did not show any significant difference in mortality of partial colectomy group when compared with TAC, the odds ratio [OR] was $1.21,95 \%$ CI 0.76 to 1.96 .

Contrary to above studies, our study included relatively recent NSQIP data set and used propensity-matched analysis, which is better modality for observational study [10]. Our results showed 81\% of patients underwent TAC as recommended by the WSES [16]. Approximately 19\% of patients underwent partial colectomy. The reasons for lower compliance with WSES were not available. There is a possibility that in certain cases, the point of care surgeon made the decision to perform partial colectomy was based on findings observed during the operation. Patients who underwent partial colectomy showed no difference in 30 days mortality $(30.1 \%$ vs. $30.1 \%)$ when compared with TAC. Our mortality was little lower than the published report [8]. The reason may be that we used the most recent dataset that may have reflected the better 
Table 1 Comparison of characteristics of patients between the two groups (TAC versus partial colectomy) before propensity matching

\begin{tabular}{|c|c|c|c|c|}
\hline Variable & $\begin{array}{l}\text { All Patients } \\
\mathrm{N}=491\end{array}$ & $\begin{array}{l}\text { Total Abdominal } \\
\text { Colectomy } \\
\mathrm{N}=398\end{array}$ & $\begin{array}{l}\text { Partial Colectomy } \\
\mathrm{N}=93\end{array}$ & $P$ value \\
\hline Age (years), Median [Q1-Q3] & $67[57-76]$ & $67[58-75]$ & $66[55-75]$ & 0.31 \\
\hline Gender & & & & 0.22 \\
\hline Female & $263(53.6)$ & $219(55)$ & $44(47.3)$ & \\
\hline Male & $228(46.4)$ & $179(45)$ & $49(52.7)$ & \\
\hline Admitted from & & & & 0.502 \\
\hline From acute care hospital inpatient & $95(19.3)$ & $82(20.6)$ & $13(14)$ & \\
\hline Not transferred (admitted from home) & $275(56)$ & $215(54)$ & $60(64.5)$ & \\
\hline Nursing home-Chronic care-Intermediate care & $75(15.3)$ & $61(15.3)$ & $14(15.1)$ & \\
\hline Outside emergency department & $38(7.7)$ & $33(8.3)$ & $5(5.4)$ & \\
\hline Transfer from other & $6(1.2)$ & $5(1.3)$ & $1(1.1)$ & \\
\hline Unknown & $2(0.4)$ & $2(0.5)$ & $0(0)$ & \\
\hline Race, white, $n(\%)$ & $341(69.5)$ & $269(67.6)$ & $72(77.4)$ & 0.084 \\
\hline Sepsis status, n (\%) & & & & 0.031 \\
\hline None & $35(7.1)$ & $28(7)$ & $7(7.5)$ & \\
\hline Sepsis & $111(22.6)$ & $80(20.1)$ & $31(33.3)$ & \\
\hline Septic Shock & $319(65)$ & $270(67.8)$ & $49(52.7)$ & \\
\hline SIRS & $26(5.3)$ & $20(5)$ & $6(6.5)$ & \\
\hline WBC count, $10^{9} / \mathrm{L}, n(\%)$ & & & & $<0.001$ \\
\hline $4-11.9$ & $97(19.8)$ & $72(18.1)$ & $25(26.9)$ & \\
\hline $12-19.9$ & $85(17.3)$ & $57(14.4)$ & $28(30.1)$ & \\
\hline $20-34.9$ & $103(21)$ & $89(22.4)$ & $14(15.1)$ & \\
\hline $35-44.9$ & $56(11.4)$ & $52(13.1)$ & $4(4.3)$ & \\
\hline$\geq 45$ & $51(10.4)$ & $41(10.3)$ & $10(10.8)$ & \\
\hline$<4$ & $34(6.9)$ & $24(6)$ & $10(10.8)$ & \\
\hline Unknown & $64(13.1)$ & $62(15.6)$ & $2(2.2)$ & \\
\hline Diabetes, $n$ (\%) & & & & 0.547 \\
\hline INSULIN & $64(13)$ & $51(12.8)$ & $13(14)$ & \\
\hline NO & $387(78.8)$ & $312(78.4)$ & 75 (80.6) & \\
\hline NON-INSULIN & $40(8.1)$ & $35(8.8)$ & $5(5.4)$ & \\
\hline Smoking, $n(\%)$ & & & & 0.724 \\
\hline No & $379(77.2)$ & $309(77.6)$ & $70(75.3)$ & \\
\hline Yes & $112(22.8)$ & $89(22.4)$ & $23(24.7)$ & \\
\hline Ventilator dependency ${ }^{\mathrm{a}}, \mathrm{n}(\%)$ & & & & 0.384 \\
\hline No & $327(66.6)$ & $261(65.6)$ & $66(71)$ & \\
\hline Yes & $164(33.4)$ & $137(34.4)$ & $27(29)$ & \\
\hline COPD, $n(\%)$ & & & & 0.387 \\
\hline No & $371(75.6)$ & $297(74.6)$ & $74(79.6)$ & \\
\hline Yes & $120(24.4)$ & $101(25.4)$ & $19(20.4)$ & \\
\hline Ascites, $n(\%)$ & & & & 0.985 \\
\hline No & $430(87.6)$ & $348(87.4)$ & $82(88.2)$ & \\
\hline Yes & $61(12.4)$ & $50(12.6)$ & $11(11.8)$ & \\
\hline $\mathrm{CHF}, n(\%)$ & & & & 0.764 \\
\hline No & $442(90)$ & $357(89.7)$ & $85(91.4)$ & \\
\hline Yes & $49(10)$ & $41(10.3)$ & $8(8.6)$ & \\
\hline HTN, $n(\%)$ & & & & 0.721 \\
\hline No & $190(38.7)$ & $152(38.2)$ & $38(40.9)$ & \\
\hline Yes & $301(61.3)$ & $246(61.8)$ & $55(59.1)$ & \\
\hline
\end{tabular}


Table 1 (continued)

\begin{tabular}{|c|c|c|c|c|}
\hline Variable & $\begin{array}{l}\text { All Patients } \\
\mathrm{N}=491\end{array}$ & $\begin{array}{l}\text { Total Abdominal } \\
\text { Colectomy } \\
\mathrm{N}=398\end{array}$ & $\begin{array}{l}\text { Partial Colectomy } \\
\mathrm{N}=93\end{array}$ & $P$ value \\
\hline CRF, $n(\%)$ & & & & 0.226 \\
\hline No & $398(81.1)$ & $318(79.9)$ & $80(86)$ & \\
\hline Yes & $93(18.9)$ & $80(20.1)$ & $13(14)$ & \\
\hline CRF on dialysis, $n(\%)$ & & & & 0.85 \\
\hline No & $422(85.9)$ & $341(85.7)$ & $81(87.1)$ & \\
\hline Yes & $69(14.1)$ & $57(14.3)$ & $12(12.9)$ & \\
\hline Disseminated cancer, $n(\%)$ & & & & 0.002 \\
\hline No & $452(92.1)$ & $374(94)$ & $78(83.9)$ & \\
\hline Yes & $39(7.9)$ & $24(6)$ & $15(16.1)$ & \\
\hline Steroid, $n(\%)$ & & & & 0.047 \\
\hline No & $388(79)$ & $307(77.1)$ & $81(87.1)$ & \\
\hline Yes & $103(21)$ & $91(22.9)$ & $12(12.9)$ & \\
\hline Weight loss & & & & 0.825 \\
\hline No & $454(92.5)$ & $367(92.2)$ & $87(93.5)$ & \\
\hline Yes & $37(7.5)$ & $31(7.8)$ & $6(6.5)$ & \\
\hline Coagulopathy, n (\%) & & & & 0.57 \\
\hline No & $388(79)$ & $312(78.4)$ & $76(81.7)$ & \\
\hline Yes & $103(21)$ & $86(21.6)$ & $17(18.3)$ & \\
\hline Blood transfusion ${ }^{\mathrm{a}}, n(\%)$ & & & & 0.525 \\
\hline No & $437(89)$ & $352(88.4)$ & $85(91.4)$ & \\
\hline Yes & $54(11)$ & $46(11.6)$ & $8(8.6)$ & \\
\hline Wound class, $n(\%)$ & & & & $<0.001$ \\
\hline 1-Clean & $3(0.6)$ & $1(0.3)$ & $2(2.2)$ & \\
\hline 2-Clean/Contaminated & $91(18.5)$ & $82(20.6)$ & $9(9.7)$ & \\
\hline 3-Contaminated & $166(33.8)$ & $141(35.4)$ & $25(26.9)$ & \\
\hline 4-Dirty/Infected & $231(47)$ & $174(43.7)$ & $57(61.3)$ & \\
\hline ASA class, $n(\%)$ & & & & 0.029 \\
\hline 2-Mild Disturb & $6(1.2)$ & $3(0.8)$ & $3(3.2)$ & \\
\hline 3-Severe Disturb & 75 (15.3) & $56(14.1)$ & $19(20.4)$ & \\
\hline 4-Life Threat & $319(65)$ & $264(66.3)$ & 55 (59.1) & \\
\hline 5-Moribund & $90(18.3)$ & 75 (18.8) & $15(16.1)$ & \\
\hline None assigned & $1(0.2)$ & $0(0)$ & $1(1.1)$ & \\
\hline
\end{tabular}

ASA; American Society of Anesthesiology, CHF; Congestive heart failure, CRF; chronic renal failure,

COPD; chronic obstructive pulmonary disease, Q1-Q3 ; first quartile-third quartile, interquartile range (IQR), SIRS; systemic inflammatory response syndrome. WBCs; White blood cell counts

a Prior to surgery

n; number of patients, \%; percentage

selection of patients to critical care management and aggressive treatment of the FCDC [19]. The other reason for lower mortality in our study could be the inclusion of all comorbidities in our propensity-matching model that can influence the post-operative mortality [20]. Our study did not find any significant difference in median hospital length of stay and 30-day post-operative complications regardless of the type of surgery was performed (TAC vs. partial colectomy). Our study added one more outcome to evaluate the discharged disposition to home and found no significant difference between the TAC vs. partial colectomy Table 3.

\section{Limitation}

The study was done from the NSQIP database; however, the database lacks the detailed information of the some of the patients' characteristics, the timing of the contraction of the clostridium difficile colitis, progression to FCDC and the timing of the colectomy from the time of identification of the FCDC. We used the most recommended 
Table 2 Comparison of characteristics of patients between the two groups (TAC versus partial colectomy) after propensity matching

\begin{tabular}{|c|c|c|c|c|}
\hline Variable & All Patients, $n=186$ & $\begin{array}{l}\text { Total Abdominal } \\
\text { Colectomy, } n=93\end{array}$ & $\begin{array}{l}\text { Partial Colectomy, } \\
n=93\end{array}$ & P-Value \\
\hline Age (years), Median [Q1-Q3] & & $65[57-75]$ & $66[55-75]$ & 0.71 \\
\hline Gender & & & & 0.755 \\
\hline Female & $91(48.9)$ & $47(50.5)$ & $44(47.3)$ & \\
\hline Male & $95(51.1)$ & $46(49.5)$ & $49(52.7)$ & \\
\hline Admitted from & & & & NA \\
\hline From acute care hospital inpatient & $33(17.7)$ & $20(21.5)$ & $13(14)$ & \\
\hline Not transferred (admitted from home) & $111(59.7)$ & $51(54.8)$ & $60(64.5)$ & \\
\hline Nursing home_Chronic care_-Intermediate care & $25(13.4)$ & $11(11.8)$ & $14(15.1)$ & \\
\hline Outside emergency department & $10(5.4)$ & $5(5.4)$ & $5(5.4)$ & \\
\hline Transfer from other & $5(2.7)$ & $4(4.3)$ & $1(1.1)$ & \\
\hline Unknown & $2(1.1)$ & $2(2.2)$ & $0(0)$ & \\
\hline Race, white, $n(\%)$ & $140(75.3)$ & $68(73.1)$ & $72(77.4)$ & 0.596 \\
\hline Sepsis status ${ }^{a}, n(\%)$ & & & & 0.956 \\
\hline None & $13(7)$ & $6(6.5)$ & $7(7.5)$ & \\
\hline Sepsis & $60(32.3)$ & $29(31.2)$ & $31(33.3)$ & \\
\hline Septic Shock & $101(54.3)$ & $52(55.9)$ & $49(52.7)$ & \\
\hline SIRS & $12(6.5)$ & $6(6.5)$ & $6(6.5)$ & \\
\hline WBC count, $10^{9} / \mathrm{L}, n(\%)$ & & & & NA \\
\hline $4-11.9$ & $45(24.2)$ & $20(21.5)$ & $25(26.9)$ & \\
\hline $12-19.9$ & $43(23.1)$ & $15(16.1)$ & $28(30.1)$ & \\
\hline $20-34.9$ & $37(19.9)$ & $23(24.7)$ & $14(15.1)$ & \\
\hline $35-44.9$ & $11(5.9)$ & $7(7.5)$ & $4(4.3)$ & \\
\hline$\geq 45$ & $15(8.1)$ & $5(5.4)$ & $10(10.8)$ & \\
\hline$<4$ & $19(10.2)$ & $9(9.7)$ & $10(10.8)$ & \\
\hline Unknown & $16(8.6)$ & $14(15.1)$ & $2(2.2)$ & \\
\hline Diabetes, $n(\%)$ & & & & 0.878 \\
\hline INSULIN & $28(15.1)$ & $15(16.1)$ & $13(14)$ & \\
\hline NO & $149(80.1)$ & 74 (79.6) & 75 (80.6) & \\
\hline NON-INSULIN & $9(4.8)$ & $4(4.3)$ & $5(5.4)$ & \\
\hline Smoking, $n(\%)$ & & & & 0.86 \\
\hline No & $142(76.3)$ & $72(77.4)$ & $70(75.3)$ & \\
\hline Yes & $44(23.7)$ & $21(22.6)$ & $23(24.7)$ & \\
\hline Ventilator dependency ${ }^{\mathrm{a}}, n(\%)$ & & & & 0.268 \\
\hline No & $124(66.7)$ & $58(62.4)$ & $66(71)$ & \\
\hline Yes & $62(33.3)$ & $35(37.6)$ & $27(29)$ & \\
\hline COPD, $n(\%)$ & & & & 0.458 \\
\hline No & $153(82.3)$ & 79 (84.9) & $74(79.6)$ & \\
\hline Yes & $33(17.7)$ & $14(15.1)$ & $19(20.4)$ & \\
\hline Ascites, $n(\%)$ & & & & 0.831 \\
\hline No & $162(87.1)$ & $80(86)$ & $82(88.2)$ & \\
\hline Yes & $24(12.9)$ & $13(14)$ & $11(11.8)$ & \\
\hline $\mathrm{CHF}, n(\%)$ & & & & $>0.99$ \\
\hline No & $169(90.9)$ & $84(90.3)$ & $85(91.4)$ & \\
\hline Yes & $17(9.1)$ & $9(9.7)$ & $8(8.6)$ & \\
\hline HTN, $n(\%)$ & & & & 0.551 \\
\hline No & $71(38.2)$ & $33(35.5)$ & $38(40.9)$ & \\
\hline Yes & $115(61.8)$ & $60(64.5)$ & $55(59.1)$ & \\
\hline $\mathrm{CRF}, n(\%)$ & & & & $>0.99$ \\
\hline No & $160(86)$ & $80(86)$ & $80(86)$ & \\
\hline
\end{tabular}


Table 2 (continued)

\begin{tabular}{|c|c|c|c|c|}
\hline Variable & All Patients, $n=186$ & $\begin{array}{l}\text { Total Abdominal } \\
\text { Colectomy, } n=93\end{array}$ & $\begin{array}{l}\text { Partial Colectomy, } \\
\mathrm{n}=93\end{array}$ & P-Value \\
\hline Yes & $26(14)$ & $13(14)$ & $13(14)$ & \\
\hline CRF on dialysis, $n(\%)$ & & & & 0.838 \\
\hline No & $160(86)$ & $79(84.9)$ & $81(87.1)$ & \\
\hline Yes & $26(14)$ & $14(15.1)$ & $12(12.9)$ & \\
\hline Disseminated Cancer, n (\%) & & & & $>0.99$ \\
\hline No & $157(84.4)$ & $79(84.9)$ & 78 (83.9) & \\
\hline Yes & $29(15.6)$ & $14(15.1)$ & $15(16.1)$ & \\
\hline Steroid, $n(\%)$ & & & & 0.814 \\
\hline No & $164(88.2)$ & $83(89.2)$ & $81(87.1)$ & \\
\hline Yes & $22(11.8)$ & $10(10.8)$ & $12(12.9)$ & \\
\hline Weight loss & & & & 0.752 \\
\hline No & $176(94.6)$ & $89(95.7)$ & $87(93.5)$ & \\
\hline Yes & $10(5.4)$ & $4(4.3)$ & $6(6.5)$ & \\
\hline Coagulopathy, n (\%) & & & & $>0.99$ \\
\hline No & $152(81.7)$ & $76(81.7)$ & $76(81.7)$ & \\
\hline Yes & $34(18.3)$ & $17(18.3)$ & $17(18.3)$ & \\
\hline Blood transfusion ${ }^{\mathrm{a}}, n(\%)$ & & & & 0.789 \\
\hline No & $168(90.3)$ & $83(89.2)$ & $85(91.4)$ & \\
\hline Yes & $18(9.7)$ & $10(10.8)$ & $8(8.6)$ & \\
\hline Wound class, n (\%) & & & & NA \\
\hline 1-Clean & $2(1.1)$ & $0(0)$ & $2(2.2)$ & \\
\hline 2-Clean/Contaminated & $29(15.6)$ & $20(21.5)$ & $9(9.7)$ & \\
\hline 3-Contaminated & $56(30.1)$ & $31(33.3)$ & $25(26.9)$ & \\
\hline 4-Dirty/Infected & $99(53.2)$ & $42(45.2)$ & $57(61.3)$ & \\
\hline ASA class, $n(\%)$ & & & & NA \\
\hline 2-Mild Disturb & $5(2.7)$ & $2(2.2)$ & $3(3.2)$ & \\
\hline 3-Severe Disturb & $33(17.7)$ & $14(15.1)$ & $19(20.4)$ & \\
\hline 4-Life Threat & $112(60.2)$ & $57(61.3)$ & $55(59.1)$ & \\
\hline 5-Moribund & 35 (18.8) & $20(21.5)$ & $15(16.1)$ & \\
\hline None assigned & $1(0.5)$ & $0(0)$ & $1(1.1)$ & \\
\hline
\end{tabular}

ASA; American Society of Anesthesiology, CHF; Congestive heart failure, CRF; chronic renal failure,

COPD; chronic obstructive pulmonary disease, Q1-Q3; first quartile-third quartile, interquartile range (IQR), SIRS; systemic inflammatory response syndrome. WBCs; White blood cell counts

a Prior to surgery

n; number of patients, \%; percentage

NA; not applicable

Table 3 Mortality and hospital length of stay between the two groups, TAC versus partial colectomy in matched data set

\begin{tabular}{llll}
\hline Variable & All patients, $\boldsymbol{n = 1 8 6}$ & $\begin{array}{l}\text { Total abdominal colectomy, } \\
\boldsymbol{n}=\mathbf{9 3}\end{array}$ & Partial colectomy, $\mathbf{n = 9 3} \quad \boldsymbol{P}$ value \\
\hline $\begin{array}{l}\text { Mortality } \\
\quad \text { Survived }\end{array}$ & $130(69.9)$ & $65(69.9)$ & $65(69.9)$ \\
$\quad$ Died & $56(30.1)$ & $28(30.1)$ & $28(30.1)$ \\
Hospital length of stay (days), & & $23[19-31]$ & $21[17-25]$ \\
Median [95\% Cl] & & \\
\hline
\end{tabular}


Table 4 Post-operative complications between the groups in matched data

\begin{tabular}{|c|c|c|c|c|}
\hline Variable & All patients, $n=186$ & $\begin{array}{l}\text { Total abdominal colectomy, } \\
n=93\end{array}$ & Partial colectomy, $N=93$ & $P$ value \\
\hline Superficial SSI & & & & $>0.99$ \\
\hline No & $182(97.8)$ & $91(97.8)$ & $91(97.8)$ & \\
\hline Yes & $4(2.2)$ & $2(2.2)$ & $2(2.2)$ & \\
\hline Deep Incisional SSI & & & & NA \\
\hline Yes & $2(1.1)$ & $0(0)$ & $2(2.2)$ & \\
\hline No & $184(98.9)$ & $93(100)$ & $91(97.8)$ & \\
\hline Organ/Space SSI & & & & $>0.99$ \\
\hline No & $171(91.9)$ & $85(91.4)$ & $86(92.5)$ & \\
\hline Yes & $15(8.1)$ & $8(8.6)$ & $7(7.5)$ & \\
\hline Wound Disruption & & & & 0.343 \\
\hline No & $176(94.6)$ & $90(96.8)$ & $86(92.5)$ & \\
\hline Yes & $10(5.4)$ & $3(3.2)$ & $7(7.5)$ & \\
\hline Pneumonia & & & & 0.176 \\
\hline No & $145(78)$ & $68(73.1)$ & $77(82.8)$ & \\
\hline Yes & $41(22)$ & $25(26.9)$ & $16(17.2)$ & \\
\hline Unplanned intubation & & & & $>0.99$ \\
\hline No & $159(85.5)$ & $80(86)$ & $79(84.9)$ & \\
\hline Yes & $27(14.5)$ & $13(14)$ & $14(15.1)$ & \\
\hline Pulmonary embolism & & & & 0.617 \\
\hline No & $182(97.8)$ & $90(96.8)$ & $92(98.9)$ & \\
\hline Yes & $4(2.2)$ & $3(3.2)$ & $1(1.1)$ & \\
\hline Ventilator dependency ${ }^{a}$ & & & & 0.766 \\
\hline No & $91(48.9)$ & $47(50.5)$ & $44(47.3)$ & \\
\hline Yes & $95(51.1)$ & $46(49.5)$ & $49(52.7)$ & \\
\hline Acute renal failure & & & & 0.814 \\
\hline Yes & $18(9.7)$ & $10(10.8)$ & $8(8.6)$ & \\
\hline No & $168(90.3)$ & $83(89.2)$ & $85(91.4)$ & \\
\hline UTI & & & & $>0.99$ \\
\hline No & $177(95.2)$ & $89(95.7)$ & $88(94.6)$ & \\
\hline Yes & $9(4.8)$ & $4(4.3)$ & $5(5.4)$ & \\
\hline CVA & & & & $>0.99$ \\
\hline No & $183(98.4)$ & $92(98.9)$ & $91(97.8)$ & \\
\hline Yes & $3(1.6)$ & $1(1.1)$ & $2(2.2)$ & \\
\hline Cardiac arrest requiring CPR & & & & 0.096 \\
\hline Yes & $17(9.1)$ & $12(12.9)$ & $5(5.4)$ & \\
\hline No & $169(90.9)$ & $81(87.1)$ & $88(94.6)$ & \\
\hline $\mathrm{Ml}$ & & & & 0.371 \\
\hline Yes & $5(2.7)$ & $1(1.1)$ & $4(4.3)$ & \\
\hline No & $181(97.3)$ & $92(98.9)$ & $89(95.7)$ & \\
\hline Blood transfusion ${ }^{a}$ & & & & 0.888 \\
\hline No & $82(44.1)$ & $42(45.2)$ & $40(43)$ & \\
\hline Yes & $104(55.9)$ & $51(54.8)$ & $53(57)$ & \\
\hline DVT & & & & NA \\
\hline Yes & $13(7)$ & $1(6.5)$ & $7(7.5)$ & \\
\hline No & $173(93)$ & $87(93.5)$ & $86(92.5)$ & \\
\hline Sepsis $^{\mathrm{a}}$ & & & & $>0.99$ \\
\hline No & $168(90.3)$ & $84(90.3)$ & $84(90.3)$ & \\
\hline Yes & $18(9.7)$ & $9(9.7)$ & $9(9.7)$ & \\
\hline Septic shock ${ }^{a}$ & & & & 0.551 \\
\hline
\end{tabular}


Table 4 (continued)

\begin{tabular}{|c|c|c|c|c|}
\hline Variable & All patients, $n=186$ & $\begin{array}{l}\text { Total abdominal colectomy, } \\
n=93\end{array}$ & Partial colectomy, $N=93$ & $P$ value \\
\hline No & $109(58.6)$ & $57(61.3)$ & $52(55.9)$ & \\
\hline Yes & 77 (41.4) & $36(38.7)$ & $41(44.1)$ & \\
\hline Return to OR & & & & $>0.99$ \\
\hline No & 163 (87.6) & $81(87.1)$ & $82(88.2)$ & \\
\hline Yes & $23(12.4)$ & $12(12.9)$ & $11(11.8)$ & \\
\hline Readmission & & & & 0.169 \\
\hline No & $167(89.8)$ & 87 (93.5) & $80(86)$ & \\
\hline Yes & $19(10.2)$ & $6(6.5)$ & $13(14)$ & \\
\hline
\end{tabular}

SSI, surgical site infection; UTI, urinary tract infection; CVA, cerebrovascular accidents; MI, Myocardial infarction; DVT, deep vein thrombosis

${ }^{\text {a }}$ Post-operative

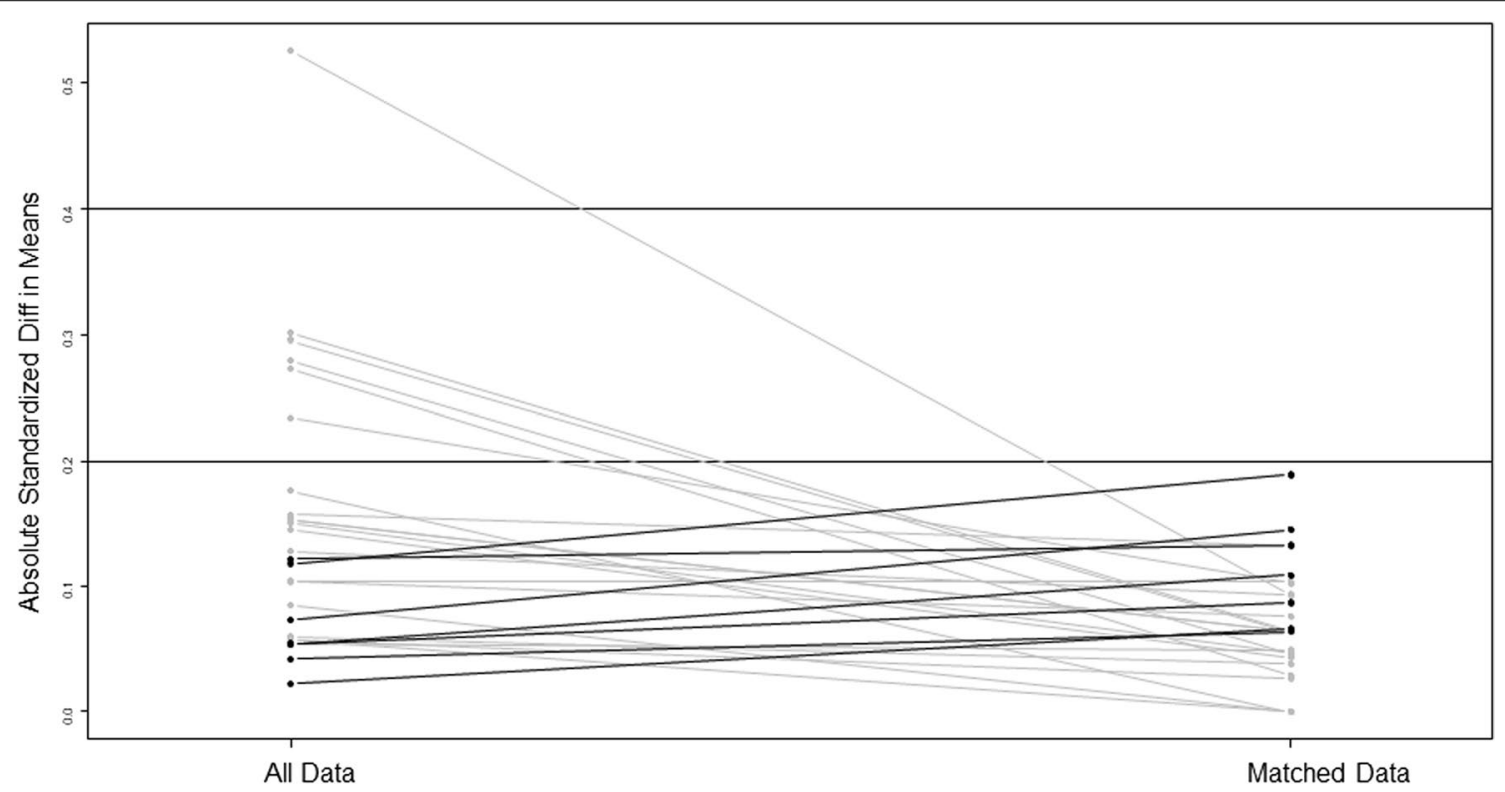

Fig. 1 Showing the improvement in standardized mean differences in variables after propensity matching

analysis method of observational study, the propensity score matching. However, that method does not take into account any unobserved or unmeasured variables that may have influenced the results.

\section{Conclusion}

The surgical mortality of FCDC remains high. Total abdominal colectomy was the procedure of choice and adapted by majority of surgeons. Partial colectomy did not increase the risk of 30 days mortality or morbidity. The discharge disposition of patients to home or rehabilitation were same regardless of the patient underwent TAC or partial colectomy.
Implications. If the disease pathology limited to one area of the colon, partial colectomy can be an alternative procedure for the FCDC patients.

\section{Abbreviations}

FCDC: Fulminant Clostridium Difficile Colitis; TAC: The Total Abdominal Colectomy; NSQIP: The National Surgical Quality Improvement Program; LOS: Length of stay; Cl: Confidence interval; LI: Loop ileostomy; PCR: Polymerase chain reaction; EIA: Enzyme immunoassays; ASA: American Society of Anesthesiologists; DM: Diabetes mellitus; COPD: Chronic obstructive pulmonary disease; HTN: Hypertension on medication; CRF: Chronic renal failure; CRF-D: Chronic renal failure on dialysis; WSES: World Society of Emergency Surgery.

\section{Acknowledgements}

Elli Gourna Paleoudis, MS, PhD performed the critical reading and final editing of the manuscript. 


\section{Authors' contributions}

Nasim Ahmed (NA) conceived and designed the study. NA was responsible for retrieving the study data, while Yen-Hong Kuo (Y-HK) performed the data analysis. NA \& Y-HK, contributed to manuscript writing. NA designed the study, accessed the data, and contributed to the manuscript. Y-HK performed the statistical analyses and contributed to the creation of manuscript. NA \& Y-HK interpreted the data. NA is also responsible for overall integrity of the study. All authors read and approved the final manuscript.

\section{Funding}

There was no funding provided for this study.

\section{Availability of data and materials}

Data are available from the American College of Surgeons.

\section{Declarations}

\section{Consent for publication}

Since the study was performed from the de-identified dataset and a retrospective study. consent from the subject were not applicable.

\section{Competing interests}

All authors declare no conflict of interest.

\section{Author details}

${ }^{1}$ Division of Trauma \& Surgical Critical Care, Jersey Shore University Medical Center, 1945 State Route 33, Neptune, NJ 07754, USA. ${ }^{2}$ Office of Research Administration, Jersey Shore University Medical Center, Neptune, NJ, USA. ${ }^{3}$ Hackensack Meridian School of Medicine, Nutley, NJ, USA.

Received: 18 November 2021 Accepted: 25 January 2022 Published online: 13 February 2022

\section{References}

1. Loo VG, Poirier L, Miller MA, et al. A predominantly clonal multi-institutional outbreak of Clostridium difficile-associated diarrhea with high morbidity and mortality. N Engl J Med. 2005;353(23):2442-9.

2. Dallal RM, Harbrecht BG, Boujoukas AJ, Sirio CA, Farkas LM, Lee KK, Simmons RL. Fulminant Clostridium difficile: an underappreciated and increasing cause of death and complications. Ann Surg. 2002;235(3):363-72.

3. Ahmed N, Kuo YH. Early colectomy safes lives in toxic mega-colon due to Clostridium difficile infection. Southern Med J. 2020;113(7):345-9.

4. Stewart D, Hollenbeak C, Wilson M. Is colectomy for fulminant Clostridium difficile colitis life saving? A systematic review. Colorectal Dis. 2013;15:798-804.

5. Napolitano LM, Edmiston CE Jr. Clostridium difficile disease: diagnosis, pathogenesis, and treatment update. Surgery. 2017;162(2):325-48.

6. Neal M, Alverdy J, Hall D, et al. Diverting loop ileostomy and colonic lavage: an alternative to total abdominal colectomy for the treatment of severe, complicated Clostridium difficile infections. Ann Surg. 2011:254:423-9.

7. Fashandi AZ, Martin AN, Wang PT, Hedrick TL, Friel CM, Smith PW, Hays RA, Hallowell PT. An institutional comparison of total abdominal colectomy and diverting loop ileostomy and colonic lavage in the treatment of severe, complicated Clostridium difficile infections. Am J Surg. 2017;213(3):507-11. https://doi.org/10.1016/j.amjsurg.2016.11.036.

8. Peprah D, Chiu AS, Jean RA, Pei KY. Comparison of outcomes between total abdominal and partial colectomy for the management of severe, complicated Clostridium difficile infection. J Am Coll Surg. 2019;228(6):925-30. https://doi.org/10.1016/j.jamcollsurg.2018.11.015.

9. Christl SU, Scheppach W. Metabolic consequences of total colectomy. Scand J Gastroenterol Suppl. 1997;222:20-4. https://doi.org/10.1080/ 00365521.1997 .11720712 .

10. Austin PC. An introduction to propensity score methods for reducing the effects of confounding in observational studies. Multivar Behav Res. 2011;46(3):399-424. https://doi.org/10.1080/00273171.2011.568786.

11. https://www.facs.org/quality-programs/acs-nsqip, Access date September 3, 2020
12. Ho DE, Imai K, King G, Stuart EA. Matchlt: nonparametric preprocessing for parametric causal inference. J Stat Softw. 2011;42(8):1-28.

13. R Core Team (2020). R: A language and environment for statistical computing. R Foundation for Statistical Computing, Vienna, Austria. https:// www.R-project.org/.

14. Sailhamer EA, Carson K, Chang Y, et al. Fulminant Clostridium difficile colitis: patterns of care and predictors of mortality. Arch Surg. 2009;144(5):433-9. https://doi.org/10.1001/archsurg.2009.51.

15. Sartelli M, Malangoni MA, Abu-Zidan FM, et al. WSES guidelines for management of Clostridium difficile infection in surgical patients. World J Emerg Surg. 2015;10:38. https://doi.org/10.1186/s13017-015-0033-6.

16. Sartelli M, Di Bella S, MCFarland LV, et al. 2019 update of the WSES guidelines for management of Clostridioides (Clostridium) difficile infection in surgical patients. World J Emerg Surg. 2019;14:8. https://doi.org/10.1186/ s13017-019-0228-3.

17. Koss A, Clark MA, Sanders A, Morton D, Keighley MR, Goh J. The outcome of surgery in fulminant Clostridium difficile colitis. Colorectal Dis. 2005. https://doi.org/10.1111/j.1463-1318.2005.00876.x.

18. Byrn JC, Maun DC, Gingold DS, Baril DT, Ozao JJ, Divino CM. Predictors of mortality after colectomy for fulminant Clostridium difficile Colitis. Arch Surg. 2008;143(2):150-4. https://doi.org/10.1001/archsurg.2007.46.

19. Gillies MA, Harrison EM, Pearse RM, Garrioch S, Haddow C, Smyth L, Parks R, Walsh TS, Lone NI. Intensive care utilization and outcomes after highrisk surgery in Scotland: a population-based cohort study. Br J Anaesth. 2017;118(1):123-31. https://doi.org/10.1093/bja/aew396.

20. Payá-Llorente C, Martínez-López E, Sebastián-Tomás JC, et al. The impact of age and comorbidity on the postoperative outcomes after emergency surgical management of complicated intra-abdominal infections. Sci Rep. 2020;10:1631. https://doi.org/10.1038/s41598-020-58453-1.

\section{Publisher's Note}

Springer Nature remains neutral with regard to jurisdictional claims in published maps and institutional affiliations.

Ready to submit your research? Choose BMC and benefit from:

- fast, convenient online submission

- thorough peer review by experienced researchers in your field

- rapid publication on acceptance

- support for research data, including large and complex data types

- gold Open Access which fosters wider collaboration and increased citations

- maximum visibility for your research: over 100M website views per year

At BMC, research is always in progress.

Learn more biomedcentral.com/submissions 